Mots. Les langages du politique

Marc Deleplace, L'Anarchie de Mably à Proudhon (1750-1850). Histoire d'une appropriation polémique

\title{
Maurice Tournier
}

\section{(2) OpenEdition}

\section{Journals}

Édition électronique

URL : https://journals.openedition.org/mots/10693

DOI : $10.4000 /$ mots. 10693

ISSN : 1960-6001

Éditeur

ENS Éditions

\section{Édition imprimée}

Date de publication : 1 juillet 2002

Pagination : 151-154

ISBN : 2-84788-011-9

ISSN : 0243-6450

\section{Référence électronique}

Maurice Tournier, « Marc Deleplace, L'Anarchie de Mably à Proudhon (1750-1850). Histoire d'une appropriation polémique », Mots. Les langages du politique [En ligne], 69 | 2002, mis en ligne le 14 mai 2008, consulté le 23 avril 2022. URL : http://journals.openedition.org/mots/10693 ; DOl : https:// doi.org/10.4000/mots. 10693

\section{(C) ENS Éditions}


Comptes rendus

Marc DELEPLACE, L'Anarchie de Mably à Proudhon (1750-1850). Histoire d'une appropriation polémique, Lyon, ENS Éditions, 2000, 290 p.

La difficulté avec 1'" Anarchisme " - même devenu notion-concept et/ou désignant sociopolitique - c'est, souligne Michel Vovelle dans sa préface, qu'avant lui et autour de lui il y a l'anarchie. Comment séparer les termes/notions politiques de cette gangue toujours négative de l' « anarchie de terrain » ? En refusant de s'y laisser entrainer.

Reste le discours. La thèse de Marc Deleplace part de là. " Ceci n'est pas une histoire de l'anarchie », prévient l'auteur dès sa première phrase. Son projet ? Tourner autour des mots et de leurs emplois aux $18^{\mathrm{e}}$ et $19^{\mathrm{e}}$ siècles. Qui est désigné par anarchiste, fauteur d'anarchie, partisan de l'anarchie, missionnaire de l'anarchie, ami de l'anarchie, suppôt de l'anarchie, anarchie aristocratique, anarchie organisée, bandes de satellites de l'anarchie, etc. ? N'importe qui, finalement, répondrait Ferdinand Brunot : «On jette ce mot à tort et à travers à tout le monde ». Anarchiste : injure universelle pour qui veut sauver la nation, c'est-à-dire tout homme politique. Pourtant, du désignant négatif à la notion explicative, la voie se frayait déjà au cours du $18^{\mathrm{e}}$ siècle. C'est le mérite de M. Deleplace de montrer comment se formule, se stylise à l'époque un ensemble conceptuel dans le maquis des diatribes et des dénonciations. " Appropriation polémique », qui, d'une signification qui se démarque des confusions de l'usage et que suggère l'ouvrage de Mably Des Droits et Devoirs du citoyen écrit en 1753 et publié en 1789, aboutira renversée aux thèses positives du Proudhon des années 1840-1850.

La première étape de cette conceptualisation a consisté en un emploi du mot anarchie comme explicatif historique : nom donné à un état de barbarie ou à un moment de crise à partir duquel il faut (re)construire l'ordre social. Des schémas circulaires ou linéaires sont proposés, de Linguet à Billaud-Varenne, dans lesquels anarchie et anarchistes ont une place assignée. L'ouvrage de Mably, précurseur en ce domaine, expose la thèse des mutations politiques successives qui, d'un état de droit initial, aboutiraient, par des voies oligarchiques (abus des magistrats) ou anarchiques (combat des chefs), à la tyrannie, au despotisme, monocraties niant la liberté et les droits naturels. Dans cette dialectique du désordre et de l'ordre, l'histoire intervient comme preuve. Il s'agit, pour les philosophes puis pour les révolutionnaires, de montrer que la mo- 
narchie est usurpatrice du pouvoir sur la ruine de l'anarchie féodale. Des analyses remarquables conduisent le lecteur à travers les processus d'historicisation, les débats constitutionnels de la Révolution, la royauté française mise en cause dans ses origines (dilemme de Mounier : monarchie ou anarchie), puis la République faisant face à l'anarchie insurrectionnelle (dilemme de Barère : République ou Révolution). Que montre l'analyse de discours? Que l'anarchie ne saurait être que cruelle, violente, monstrueuse, funeste, déplorable, dangereuse, etc. En même temps, des fluctuations dans les emplois affectent de manière égale le discours réflexif et le discours polémique. D'où ce constat de M. Deleplace : « La corrélation que l'on peut établir entre les variations de la courbe des occurrences de l'anarchie, les fluctuations de l'équilibre interne des énoncés, la modification des proportions entre causes et acteurs, parmi les sujets de l'anarchie, assoit la valeur de dénomination politique de l'anarchie et conforte l'hypothèse de l'étroite relation entre notion-concept et désignant sociopolitique » (p. 90).

Le désignant d'anarchiste lui-même (que l'on peut dater de 1791) apparait ces années-là dans le discours girondin. L'anarchiste y a partie liée avec les figures de l'intrigant et du factieux, bientôt de l'enragé, puis tient compagnie à jacobin, montagnard, sans-culotte en attendant les buveurs de sang de «93 ». En point de mire : Marat, qui «prêche/ propage/provoque l'anarchie $»$. Le maitre-mot de ce discours est désorganisation. Les anarchistes, mot devenu plus fréquent au pluriel, sont vus comme formant un parti (ensemble de partisans, à l'époque), un coin à l'Assemblée (le coin des anarchistes, selon Brissot, p. 105). Virage discursif? Les anarchistes, à partir de 1793, sont aussi associés aux royalistes, fanatiques, prêtres voire au parti de l'étranger, l'anarchie donnant la main à la contre-révolution. Enfin, il s'agit de Robespierre en personne : l'anarchiste s'est fait terroriste.

Une très riche analyse de discours, précise, datée, comptabilisée, s'appuyant sur les associations, les oppositions, les qualifications du désignant anarchiste, sous-tend l'histoire du discours girondin et de ses fluctuations. L'analyse se poursuit sur le discours thermidorien, après retournement du référent contre les Girondins, coupables d' ' abus des mots » : les vrais anarchistes sont à trouver parmi les censeurs de l'anarchie. Bref, l'anarchiste c'est toujours l'autre. Le serment civique du 2 pluviôse an 5 (21 janvier 1797) donne le la fondamental : « Je jure haine à la royauté, haine à l'anarchie, je jure attachement et fidélité à la République... » (p. 121). L'anarchie est toujours anti-républicaine.

En pareil cas, le renversement des valeurs est difficile. Il se produit pourtant, quoique rarement : Jourdan célèbre le 14 juillet comme jour 
d'anarchie « où le peuple a reconquis ses droits ». On dirait que, pour reprendre 1'expression de M. Deleplace, le mot se dévitalise. Il en profite pour multiplier ses occurrences et aussi pour installer dans ses emplois une cohérence en relation avec despotisme : royauté $=$ despotisme d'un seul ; anarchie = despotisme de tous. Au Conseil des Cinq-cents on honnit ensemble le «despotisme de l'anarchie et de la royauté » (p. 143).

À cette définition politique s'ajoute une figure sociale, dont le réseau associatif ne parait pas avoir bougé : désordre, crime, discorde, désorganisation, trouble, confusion, mais aussi esprit de révolte, discours séditieux et, au degré suprême, guerre civile. Telle est sa « toile de fond permanente ", écrit M. Deleplace. Mais le rôle de l'anarchie ne se confine pas à la "grande peur des riches » (Soboul, p. 160). Née du droit naturel contre la propriété féodale, l'anarchie pointe sa vindicte vers toute propriété. D'où les pillards de l'an 2. D'où, en pleine disette, les lois du maximum protégeant ceux qui ne possèdent rien. D'où la « loi agraire » de Babeuf et des Égaux. L'anarchie se ferait-elle autoritaire ? Cette contradiction est encore plus visible quand on confronte anarchie - contrôle fort sur l'économie - et libéralisme - liberté absolue dans la possession et le commerce. M. Deleplace conclut avec justesse : « Par une contradiction lexicale d'une ampleur exceptionnelle, la liberté illimitée peut être à la fois synonyme d'anarchie, dans le discours politique, et antinomique de l'anarchie, dans le discours social» (p. 167). De politique donc le danger de l'anarchie est devenu social : ce sont les prédicateurs de la loi agraire qui menacent l'ordre. L'étiquette elle-même n'en est que plus négative. "L'anarchie dévore tout», s'écriait Barère en 1793. Une nouvelle image se dégage avec les crises économiques : n'est-ce pas l'anarchiste, sans-travail, sans-foi et sansbiens, qui s'incarnera dans le sous-prolétariat, « classe dangereuse »?

Pour que le désignant sociopolitique s'assume enfin, un retournement complet est nécessaire. Alors que la restauration royaliste identifie la Révolution à l'anarchie terroriste, que Chateaubriand met sur le même plan esprit de nivellement, anarchie des idées (= le romantisme ?) et démocratie (p. 216) et que même le dictionnaire républicain de GarnierPagès (1842) se fait l'écho de la doxa péjorative, Proudhon franchit le pas : "Comme l'homme cherche la justice dans l'égalité, la société cherche l'ordre dans l'anarchie » (p. 231). À côté du mot négatif, héritier des exécrations du $18^{\mathrm{e}}$ siècle, un mot nouveau se met en place dans son discours, positif, le même mais renversé. Il s'agit, pour M. Deleplace, d'une « subversion » de la notion, la liberté se remettant sous le couvert de la loi. Citation centrale : «La liberté est anarchie 
parce qu'elle n'admet pas le gouvernement de la volonté, mais seulement celui de l'autorité de la loi, c'est-à-dire de la nécessité ». Quel dommage que M. Deleplace se contente d'évoquer Maitron et Dolléans, sans soumettre les énoncés proudhoniens à la sagacité de ses analyses de discours, comme il l'avait si bien fait pour Mably, Mounier ou Brissot. Il est vrai que l'œuvre de Proudhon est si énorme - et parfois si paradoxale - qu'il y faudrait une seconde thèse, et de même facture.

Maurice Tournier

Francesca CABASINO, Formes et enjeux du débat public, Discours parlementaire et immigration, Rome, Bulzoni, 2000, 223 p.

Francesca Cabasino est professeure de français à l'Université de Rome «La Sapienza », Faculté de Sciences politiques. Elle consacre ses recherches à la pragmatique de l'énonciation et à l'argumentation dans le discours politique.

À partir de la notion de "polylogue différé », elle se propose de caractériser le débat parlementaire. «La nature particulière de l'échange parlementaire met en relation une pluralité d'acteurs, mais on ne peut pas dire qu'une véritable interaction ait lieu. En effet les participants au débat sont obligés par un règlement très contraignant de suivre un ordre d'intervention donné et de respecter le temps de parole prévu par le président au début de la première séance ${ }^{1}$. À cause de cette dislocation, le résultat est le contraire d'un dialogue spontané ». Son étude porte sur la comparaison entre deux projets de lois au Parlement français : le projet Debré présenté par la droite et le projet Chevènement, dit RESEDA (Relatif à l'Entrée et au Séjour des Étrangers et au Droit d'Asile) élaboré par la gauche à quelques mois de distance. Le corpus est sélectionné sur la base du compte rendu intégral des séances du Sénat (4 et 5 février 1997 et 21, 22 et 27 janvier 1998) et de l'Assemblée nationale ( 8 avril 1998 lors de discussions en dernière lecture) publié dans le Journal Officiel.

L'ouvrage est divisé en cinq parties. La première est constituée de rappels théoriques sur les fondements de l'analyse du discours. La

1. Le temps de parole varie selon l'importance numérique du groupe d'appartenance. Dans la discussion générale du projet de loi en question, le 4 février 1997, on passe des 71 minutes accordées au RPR, aux 62 des Socialistes et aux 28 minutes du Groupe Communiste républicain et citoyen. Le même ordre sera respecté dans la séance du 21 janvier 1998, avec de très légères modifications concernant les contraintes de temps. 